\title{
Experience in clinical diagnosis and treatment of duodenal tumors
}

\author{
ZHENG WANG $^{1,2}$, ZIHAI DING $^{1}$, SHIJIE HUANG ${ }^{3}$ and SHIZHEN ZHONG ${ }^{1}$ \\ ${ }^{1}$ Anatomical Institute of Minimally Invasive Surgery, Southern Medical University, Guangzhou, Guangdong 510515; \\ ${ }^{2}$ Department of General Surgery of Guangzhou Hospital of Integrated Traditional Chinese and Western Medicine, \\ Guangzhou, Guangdong 510800; ${ }^{3}$ Department of Gastroenterology Surgery of The 4th Affiliated Hospital of \\ Jinan University, Guangzhou Red Cross Hospital, Guangzhou, Guangdong 510220, P.R. China
}

Received March 17,2016; Accepted September 20, 2016

DOI: $10.3892 / \mathrm{mco} .2016 .1061$

\begin{abstract}
Small bowel tumors are rare tumors. Duodenal tumors occur more commonly compared with other small intestinal tumors. To summarize the clinicopathological features of duodenal tumors, in the present study 44 cases of duodenal tumors were collected, and the comparative clinicopathological characteristics between tumors of the ampulla and non-ampulla, the choice of treatment, and differences in the prognosis, were analyzed. The pathological type identified was predominantly adenocarcinoma; periampullary duodenal tumors were almost classifiable as adenocarcinoma in terms of their type. Non-ampulla duodenal tumors also included rare pathological types, such as stromal tumor and large B-cell lymphoma. The symptoms of duodenal tumors were non-specific, therefore rendering early diagnosis and treatment difficult. Due to jaundice, periampullary duodenal tumors were diagnosed earlier than non-ampulla duodenal tumors. Endoscopy and computed tomography (CT) examinations were valuable in terms of diagnosis, and were used as a means of screening. Carcinoembryonic antigen (CEA) and cancer antigen 199 (CA199) were revealed to be important as biomarkers. Radical surgery was the most effective treatment. Pancreaticoduodenectomy was revealed to be applicable in all cases of duodenal tumors. For non-ampulla duodenal tumors, partial duodenum resection and subtotal gastrectomy were appropriate for selection as methods of treatment. No survival benefits were identified for adjuvant chemotherapy. Duodenal tumors were shown to be a rare neoplasm with atypical symp-
\end{abstract}

Correspondence to: Dr Zheng Wang, Department of General Surgery of Guangzhou Hospital of Integrated Traditional Chinese and Western Medicine, 87 Yingbin Road, Huadu, Guangzhou, Guangdong 510800, P.R. China

E-mail:wzsjw@126.com

Professor Zihai Ding, Anatomical Institute of Minimally Invasive Surgery, Southern Medical University, 1838 Guangzhou North Street, Baiyun, Guangzhou, Guangdong 510515, P.R. China

E-mail: dingzih@163.com

Key words: duodenal tumor, clinopathology, diagnosis and treatment toms; they should be diagnosed and treated as early as possible; $\mathrm{CT}$ and gastroscopy may be used for screening, radical surgery offers the best treatment; pancreatoduodenectomy is not the only surgery option available; and chemotherapy did not result in any survival benefits.

\section{Introduction}

The onset of cancer may appear insidiously, and clinical symptoms of duodenal papilla tumors are not typical at the start. In digestive tract tumors, the incidence rate of small bowel tumors is $<2 \%$ (1). Duodenal neoplasms, including primary and secondary tumors, occur rarely. The incidence of duodenal malignant tumors accounted for 33-45\% of all small intestine tumors (2), and the most common duodenal lesions were identified as being adenocarcinoma (clinically, $77 \%$ of duodenal tumors) $(3,4)$. In terms of location, in the present study it was hypothesized that the clinicopathological characteristics, diagnosis and methods of treatment, comparing between ampulla and non-ampulla duodenal tumors, were likely to exhibit certain differences. In the early stages, the insidious clinical manifestation of duodenal tumors easily leads to misdiagnosis and missed diagnoses, and therefore this area warranted further investigation (5).

\section{Materials and methods}

Patients. The records of all patients with duodenal tumors treated in our hospital, i.e. Guangzhou Hospital of Integrated Traditional Chinese and Western Medicine between January 1, 1995 and December 31, 2012 were retrospectively reviewed. The diagnosis of duodenal tumors was conducted by histological examination, and confirmed by subsequent surgical pathology or endoscopic biopsy.

Data on patients' age, gender, the tumor location, symptoms, palliative check-up, cancer antigen 199 (CA199) and carcinoembryonic antigen (CEA) biomarkers, treatment, tumor stage and survival outcome were collected. Treatment data included the type of resection and adjuvant treatment, and supportive therapy. The patients' records/information was anonymized and de-identified prior to the analysis.

The study protocol conformed to the ethical guidelines of the 1975 Declaration of Helsinki (6th revision, 2008), as reflected in a priori approval by the Medical Ethics Committee 
of Guangzhou Hospital of Integrated Traditional Chinese and Western Medicine.

Statistical analysis. Associations between tumor location and clinicopathological variables were analyzed using the $\chi^{2}$ test. Survival curves were estimated using the Kaplan-Meier method, and compared using the log-rank test. All statistical analyses were performed with SPSS 13.0 software, version 13.0 (SPSS, Inc., Chicago, IL, USA). All tests were two-sided, and $\mathrm{P}<0.05$ was considered to indicate a statistically significant value.

\section{Results}

Patient characteristics. A total of 57 patients with small intestinal tumors were admitted for treatment to Guangzhou Hospital of Integrated Traditional Chinese and Western Medicine between January 1, 1995 and December 31, 2012, and of these, 44 patients $(77.2 \%)$ were diagnosed with duodenal tumors, among which 24 were located in the ampulla and 20 were located in the non-ampulla (Table I). Gender distribution, age, smoking and drinking habits, and any family history of cancer revealed no significant differences $(\mathrm{P}>0.05)$ between ampulla and non-ampulla duodenal tumors (Table I). The majority of the patients were $>60$ years of age (Table II). A significant difference in the symptoms of jaundice between patients with non-ampulla and ampulla duodenal tumors was observed $(\mathrm{P}<0.05)$ (Table I), although the other clinical manifestations of the two locations did not disclose any especial differences compared with the other digestive tract tumors, which provides one of the main reasons why duodenal tumors cannot be diagnosed early.

Auxiliary examination for diagnosis. The most commonly used auxiliary examination techniques for diagnosing all duodenal tumors are gastroscopy, computed tomography (CT), ultrasound and digestive tract radiography. Abdominal plain film is a routine examination, although it is more suitable for intestinal obstructions, particularly for non-ampullary duodenal tumors. For ampulla duodenal neoplasms, magnetic resonance $(\mathrm{MR}) \backslash \mathrm{MR}$ cholangiopancreatography (MRCP) endoscopic retrograde $\mathrm{CP}(\mathrm{ERCP}) \backslash$ percutaneous transhepatic cholangial drainage (PTCD) may be useful for revealing positive findings, which is also a common examination for obstructive jaundice (Table III). Evidently, surgical pathology is the technique best suited to offering the truest diagnosis.

Role of tumor markers in diagnosis. For ampulla and non-ampulla duodenal tumors, the percentages of the high tumor markers, CA199 or CEA, reached 40-50\%, which evidently has a certain significance in terms of the diagnosis of duodenal tumor (Tables IV and V).

Pathological types of duodenal tumors. Consistently with small intestine tumors, the pathological types of the duodenal tumors were predominantly adenocarcinoma. The pathological types of ampullary duodenal tumors followed up were all adenocarcinoma; however, the non-ampullary duodenal neoplasms were mainly adenocarcinoma, with rare pathological types, including stromal tumor and large B-cell lymphoma (Table VI and Figs. 1-3).
Tumor location and pathological stage. Ampulla and non-ampulla tumors of the duodenum may be clearly assigned to pathological staging. In the analysis, neither the TNM stage nor T- staging demonstrated any obvious differences, comparing between the ampulla and non-ampulla tumors. However, pathological $\mathrm{N}$ staging and the $\mathrm{M}$ stage were late for non-ampulla tumors compared with ampulla duodenal tumors in the diagnosis, which might be associated with more atypical symptoms of non-ampulla duodenal tumors, which render an early diagnosis difficult (Table VII).

Treatment of duodenal tumors. As for other cancer types, the treatment included surgery, surgery plus chemotherapy and supportive therapy (see Table VIII). The reasons for the choice of supportive therapy were as follows: Advanced metastases unable to be operated on ( 7 cases, including 2 cases of ampulla and 5 cases of non-ampulla); patients or their families refused surgery ( 7 cases, including 3 cases of ampulla and 4 cases of non ampulla); or reason unknown (3 cases, including 1 case of ampulla and 2 cases of non ampulla).

Surgical method of duodenal tumor. Although the predominant surgical approach was pancreatoduodenectomy, a difference remained in terms of the surgical procedure for ampulla and non-ampulla duodenum tumors (Table IX). Duodenal ampulla tumor radical resections were all performed as pancreatoduodenectomy. However, for non-ampulla duodenal tumors, partial resection of the duodenum and Billroth II subtotal gastrectomy also were able to attain the purpose of radical cure resection according to the tumor location, although pancreatoduodenectomy was assigned priority. The probability of a palliative operation for duodenal ampulla cancer was also higher. As for the tumor margins, the authors consider that, as long as the tumor is removed cleanly, the spacing is not constant, although a larger sample would be required to analyze this further.

Survival of duodenal adenocarcinoma. To facilitate comparison, only the pathological types of duodenal adenocarcinoma were included for a comparison of the various treatment methods and to draw the survival curve. As shown in Table $\mathrm{X}$ and Figs. 4-7, the survival rate of patients receiving radical surgery was the best, and chemotherapy had little effect on the survival of the patients. It was unexpected that no difference would be observed in terms of the survival rate of patients receiving palliative surgery and conservative treatment, although a larger sample size would be required to substantiate these findings.

Case analysis of patients with duodenal tumors with long-term survival. A total of 20 patients underwent radical operation. A total of seven patients experienced long-term survival (over 3 years), and all of these underwent radical operation.

Fifteen cases received radical pancreatoduodenectomy, of which four cases experienced long-term survival. There were five cases of other radical operations, including three cases that experienced long-term survival.

A total of seven patients with long-term survival included three cases of duodenal ampulla adenocarcinoma (17 cases of duodenal ampulla cancer surgery, including 12 cases of radical surgery, all underwent pancreatoduodenectomy). Four cases 
Table I. General pathological features of duodenal tumors.

\begin{tabular}{|c|c|c|c|}
\hline \multirow[b]{2}{*}{ Characteristic } & \multicolumn{2}{|c|}{ Number of patients } & \multirow[b]{2}{*}{ P-value } \\
\hline & Non-ampulla & Ampulla & \\
\hline Gender & & & 1 \\
\hline Male & $9(45.0 \%)$ & $12(50.0 \%)$ & \\
\hline Female & $11(55.0 \%)$ & $12(50.0 \%)$ & \\
\hline Age, years & & & 0.813 \\
\hline$<60$ & $6(30.0 \%)$ & $8(33.3 \%)$ & \\
\hline$\geq 60$ & $14(70.0 \%)$ & $16(66.7 \%)$ & \\
\hline Alcohol consumption & & & 0.488 \\
\hline No & 17 & 22 & \\
\hline Yes & 3 & 2 & \\
\hline Smoking & & & 0.284 \\
\hline No & 15 & 21 & \\
\hline Yes & 5 & 3 & \\
\hline Family tumor history & & & 0.268 \\
\hline No & 19 & 24 & \\
\hline Yes & 1 & - & \\
\hline Clinical feature & & & 0.001 \\
\hline Bellyache & 9 & 8 & \\
\hline Jaundice & 1 & 15 & \\
\hline Bloating & 4 & 2 & \\
\hline Gastrointestinal bleeding & 3 & 2 & \\
\hline Nausea, vomiting & 6 & 2 & \\
\hline Abdominal mass & - & - & \\
\hline Anorexia & 1 & 2 & \\
\hline Acid regurgitation, belching & 3 & 1 & \\
\hline Anal stop exhaust defecation & 1 & 1 & \\
\hline Diarrhea & 1 & - & \\
\hline Other & 3 & 4 & \\
\hline No obvious features & 4 & - & \\
\hline
\end{tabular}

No significant differences in gender distribution with respect to non-ampulla and ampulla tumors were observed $(\mathrm{P}=1.0)$. The gender ratio of male to female was essentially equal. The age distribution ( $<60$ or $\geq 60$ years) did not disclose any significant differences in terms of non-ampulla and ampulla tumors $(\mathrm{P}=0.813)$, although the $\geq 60$ years of age group accounted for the majority of the patients. Habits of alcohol consumption with respect to non-ampulla and ampulla tumors: No significant difference $(\mathrm{P}=0.488)$; smoking habits: No significant difference $(\mathrm{P}=0.284)$. Any family history of tumors with respect to non-ampulla and ampulla tumors: No significant difference $(\mathrm{P}=0.268)$. Compared with other digestive system tumors, the symptoms of jaundice in the duodenal ampulla were greater than those in the non-ampulla, and this demonstrated a significant difference $(\mathrm{P}=0.001)$.

were long-term survival were associated with non-ampulla duodenal neoplasms (10 cases of duodenal non-ampulla cancer surgery, including eight cases of radical surgery), including one case of stromal tumor, one case of large B-cell lymphoma and two cases of adenocarcinoma. Only one case was operated with the operation of pancreatoduodenectomy.

If it was operable, the prognosis of the non-papillary tumor was improved, which may be associated with the long-term survival of the pathological types of non-gland cancer, which accounted for $50 \%$. The operation effect of stromal tumors was also better.

Regarding the long-term survival of seven patients, three had no option of a pancreatoduodenectomy. In fact, performing a pancreatoduodenectomy has itself also been questioned due to the difficulty of the operation, the procedure is not fully standardized because of the complexity of the operation, or the scope of the surgery or cleaning requires further improvement.

\section{Discussion}

The majority of the malignant tumors of the small intestine are located in the duodenum, which are predominantly adenocarcinoma (6). Primary duodenal adenocarcinoma is observed only rarely in the clinic, and this accounts for $\sim 43 \%$ of all small bowel adenocarcinoma (7). Differences are clearly evident in different sections of the duodenum with regard to 
Table II. Association between the patients' age and tumor location.

\begin{tabular}{lcc}
\hline & Mean age & $95 \%$ CI \\
\hline Duodenum & $67.3 \pm 16.9$ & $(59.3,75.2)$ \\
Ampulla & $66.5 \pm 12.3$ & $(61.3,71.7)$ \\
\hline
\end{tabular}

No significant difference in age of the patients was identified between those with ampulla duodenal or non-ampulla tumors $(\mathrm{P}>0.05)$. CI, confidence interval.

Table III. Auxiliary examination for diagnosis.

\begin{tabular}{lcc}
\hline & \multicolumn{2}{c}{ Number of patients } \\
\cline { 2 - 3 } & Duodenum & Ampulla \\
\hline Endoscopy & $12(60.0 \%)$ & $14(58.3 \%)$ \\
CT & $7(35.0 \%)$ & $13(54.2 \%)$ \\
Ultrasound & 2 & 8 \\
Digestive tract radiography & 3 & 7 \\
Abdominal plain film & 1 & \\
MR & & 7 \\
MRCP & & 5 \\
ERCP & & 6 \\
PTCD & & 2 \\
\hline
\end{tabular}

The techniques listed in this Table are commonly used for the examination of digestive tract diseases, although duodenal tumors provide a focus. Endoscopy and CT were used the most commonly. MRIMRCP\ERCP\PTCD are advantageous in terms of diagnosing ampulla duodenal neoplasms. CT, computed tomography; MR, magnetic resonance; MRCP, MR cholangiopancreatography; ERCP, endoscopic retrograde $\mathrm{CP}$; $\mathrm{PTCD}$, percutaneous transhepatic cholangial drainage.

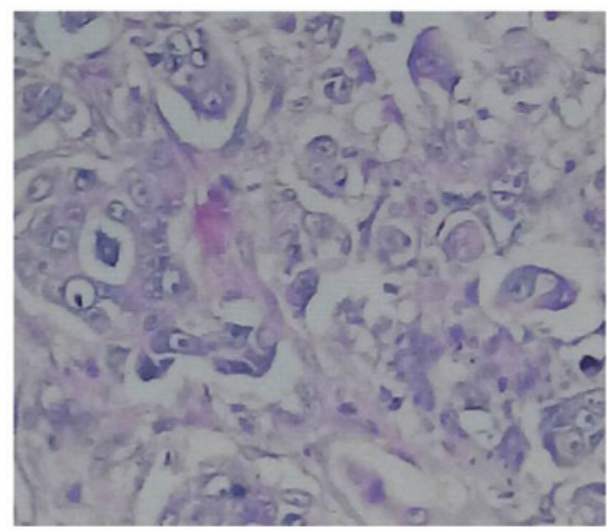

Figure 1. A representative image of adenocarcinoma of the duodenal ampulla Adenocarcinoma of duodenal ampulla II class cell differentiation is shown.

the incidence of primary duodenal adenocarcinoma, with the majority located in the area surrounding the nipple (8). The present case study suggests that duodenal adenocarcinoma

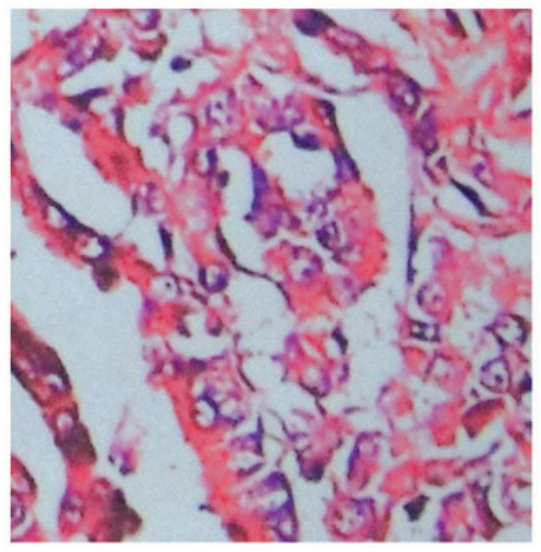

Figure 2. A representative image of adenocarcinoma of the non-ampulla duodenum. Duodenal bulb low-differentiated adenocarcinoma (II-III) is shown.

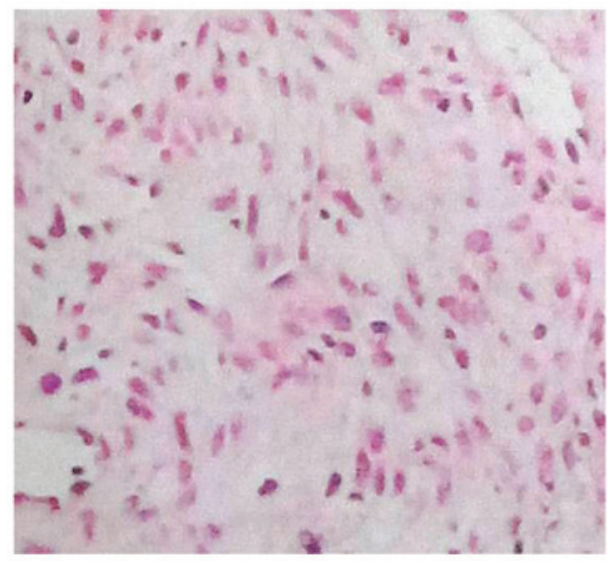

Figure 3. A representative image of a stromal tumor of non-ampulla duodenum. Gastrointestinal stromal tumor of a duodenal horizontal segment is shown.

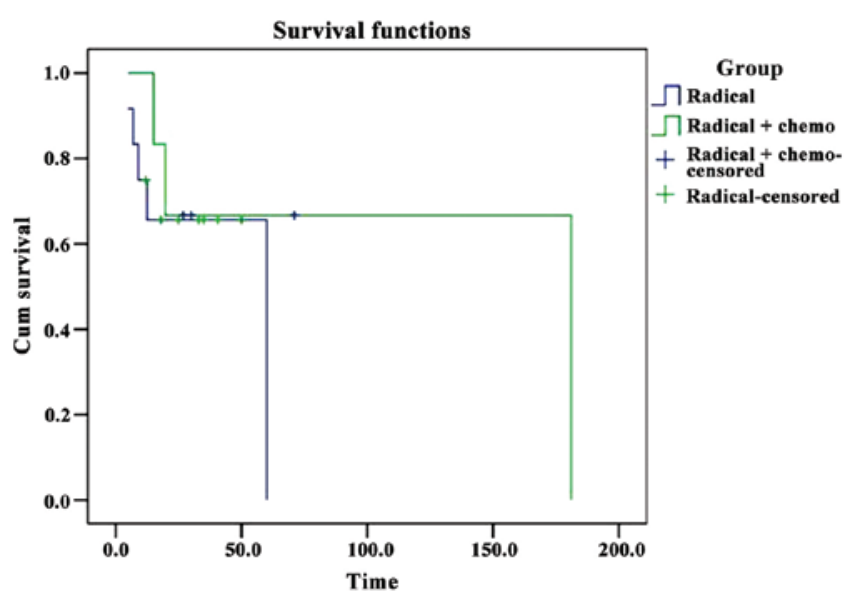

Figure 4. Kaplan-Meier curve of radical surgery (no chemotherapy) and radical surgery + chemotherapy. No significant difference in the curative effect was observed between radical surgery (no chemotherapy) and radical surgery + chemotherapy $(\mathrm{P}>0.05)$, and the median overall survival was 60.0 and 181.0 months, respectively. Chemo, chemotherapy; Cum, cumulative.

predominantly accounts for the vast majority of malignant tumors of the small intestine, slightly more so for the ampulla than the non-ampulla. 
Table IV. CA199 values of adenocarcinoma on admission (or pre-operation).

\begin{tabular}{|c|c|c|c|c|}
\hline \multirow[b]{2}{*}{ Location } & \multicolumn{3}{|c|}{ Number of patients } & \multirow[b]{2}{*}{ High CA199 ratio (\%) } \\
\hline & High CA199 & Not high CA199 & Total & \\
\hline Non-ampulla & 7 & 10 & 17 & 41.18 \\
\hline Ampulla & 12 & 12 & 24 & 50.00 \\
\hline Total & 19 & 22 & 41 & 46.34 \\
\hline
\end{tabular}

Statistical analysis: $\chi^{2}=0.312, v=1, \mathrm{P}=0.752$ (two-sided test). The difference was not significant. It may be considered that there is no difference between the CA199 index of duodenal non-ampulla and ampulla. The positive rate of CA199 was observed to be $40-50 \%$, therefore it is valuable for the diagnosis of duodenal tumors, although the sensitivity is not high. CA199, cancer antigen 199.

Table V. CEA values of adenocarcinoma on admission (or pre-operation).

\begin{tabular}{lcccc}
\hline & \multicolumn{3}{c}{ Number of patients } & \\
\cline { 2 - 4 } Location & High CEA & Not high CEA & Total & High CEA ratio (\%) \\
\hline Non-ampulla & 8 & 9 & 17 & 47.06 \\
Ampulla & 10 & 14 & 24 & 41.67 \\
Total & 18 & 23 & 41 & 43.90 \\
\hline
\end{tabular}

Statistical analysis: $\chi^{2}=0.117, v=1, \mathrm{P}=0.760$ (two-sided). The difference was not significant. It may be considered that there is no difference between the CEA index of duodenal non-ampulla and ampulla. The positive rate of CEA was observed to be $\sim 40-50 \%$, therefore it is valuable for the diagnosis of duodenal tumors, although the sensitivity is not high. CEA, carcinoembryonic antigen.

Table VI. Pathological types.

\begin{tabular}{lccc}
\hline & \multicolumn{3}{c}{ Number of patients } \\
\cline { 2 - 4 } Location & Adenocarcinoma & Stromal tumor & Large B-cell lymphoma \\
\hline Non-ampulla & $17(85 \%)$ & $2(10 \%)$ & $1(5 \%)$ \\
Ampulla & 24 & - & - \\
\hline
\end{tabular}

Statistical analysis: $\chi^{2}=3.863, v=2, \mathrm{P}=0.145$ (two-sided). The difference was not significant. No significant difference was identified between the pathological types of ampulla and non-ampulla duodenal neoplasms. The tumors of the duodenal ampulla were revealed to be adenocarcinoma; the majority of non-duodenal ampulla tumors were also adenocarcinoma.

Notably, the clinical manifestations of primary duodenal adenocarcinoma were lacking in specificity, and the incidence rate was low, therefore this may easily escape the attention of clinicians, making early diagnosis difficult. The first diagnosis easily becomes a misdiagnosis of the common digestive tract diseases, including gastritis, gallstones, and so forth. Improvements in the ability of doctors and patients to understand the disease would also help to improve vigilance, in order to assist in forming a correct, early diagnosis. The present study has also determined that, with the exception of the symptoms of jaundice in patients with tumors of the duodenal ampulla, which offer certain insights into possible diagnostic ranges, the clinical manifestations of duodenal tumors were not specific to other digestive tract diseases.
For adenocarcinomas surrounding the area of nipple, ERCP currently offers the best means of examination (9). Upper digestive tract radiography has an important use for the diagnosis of tumors of the duodenal 3 and 4 segments, which may indicate that tumors of the distal duodenum are difficult to reach using an endoscope. B-ultrasound, CT and MR imaging (MRI) are not very specific for the diagnosis of this disease, and it is not easy to differentiate bile duct stones from cancer around the ampulla. However, they are useful as early screening measures. At the same time, they are very useful as techniques in terms of tumor staging and operation mode selection. CT is recommended as the first choice for the diagnosis of primary duodenal adenocarcinoma (10). The present study has revealed that the diagnosis of duodenal tumors by endoscopy and CT 
Table VII. Evaluation of the pathological stage of adenocarcinoma.

\begin{tabular}{lccr}
\hline & \multicolumn{2}{c}{ Number of patients } & \\
\cline { 2 - 3 } & Duodenum & Ampulla & P-value \\
\hline TNM stage & & & 0.078 \\
I & & 3 & \\
II & 2 & 6 & \\
III & 2 & 2 & 0.365 \\
IV & 9 & 4 & \\
T-stage & & & \\
Tis & & & \\
T1 & & & \\
T2 & & 3 & \\
T3 & & 3 & \\
T4 & 2 & 5 & \\
N-stage & 4 & & \\
N0 & & 9 & \\
N1 & & & \\
N2 & 1 & & \\
M-stage & 2 & 5 & \\
M0 & & & \\
M1 & 3 & & \\
\hline
\end{tabular}

TNM staging and tumor location: Statistical analysis: $\chi^{2}=6.815, v=3$, $\mathrm{P}=0.078$ (two-sided). The difference was not significant. $\mathrm{T}$ staging and tumor location: $\chi^{2}=2.015, v=2, \mathrm{P}=0.365$ (two-sided). The difference was not significant. $\mathrm{N}$ staging and tumor location: $\chi^{2}=10.040$, $\nu=2, \mathrm{P}=0.007$ (two-sided). The difference was significant, with the $\mathrm{N} 2$ period for non-ampulla being more so. Also, the N0 and N1 period s for ampulla duodenal tumors were more so. $\mathrm{M}$ staging and tumor location: $\chi^{2}=4.636, v=1, \mathrm{P}=0.031$ (two-sided). The difference was significant, with the M1 period for non-ampulla being more so. Tis, tumor in situ.

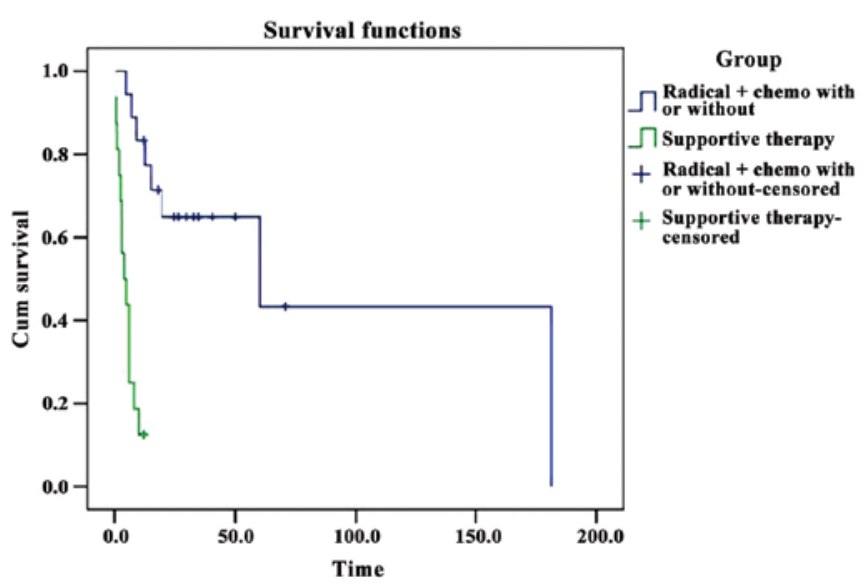

Figure 5. Kaplan-Meier curve of radical surgery (with or without chemotherapy) and supportive therapy. There was a significant difference in curative effect between radical surgery (with or without chemotherapy) and supportive therapy $(\mathrm{P}=0.05)$, and the median overall survival was 60.0 and 4.0 months, respectively. Chemo, chemotherapy; Cum, cumulative.

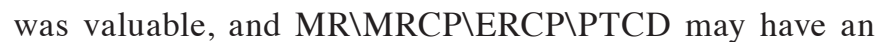
applicability for tumors of the duodenal ampulla. In addition,

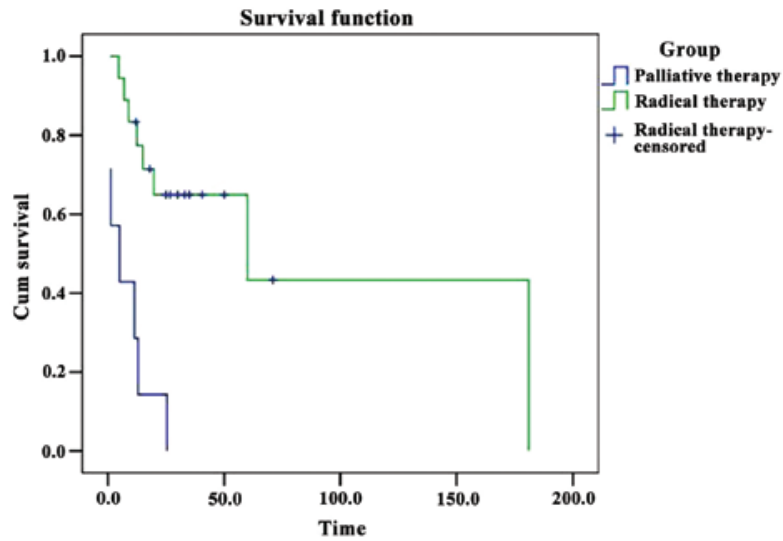

Figure 6. Kaplan-Meier curve of radical surgery (with or without chemotherapy) and palliative therapy (with or without chemotherapy). There was a significant difference in the curative effect between radical surgery (with or without chemotherapy) and palliative therapy (with or without chemotherapy) $(\mathrm{P}=0.001)$, and the median overall survival was 60.0 and 5.0 months, respectively. Cum, cumulative.

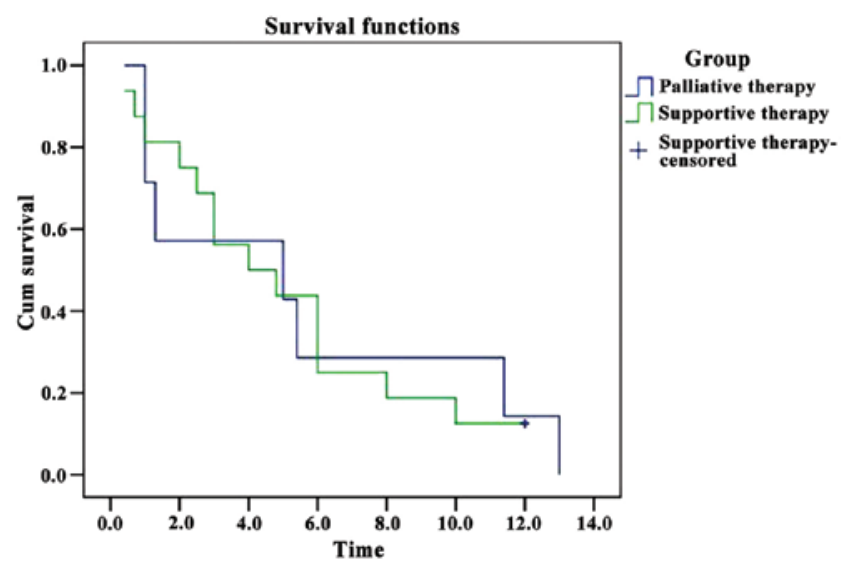

Figure 7. Kaplan-Meier curve of Palliative therapy (with or without chemotherapy) and supportive therapy. No significant difference was observed in the curative effect between palliative therapy (with or without chemotherapy) and supportive therapy $(\mathrm{P}>0.05)$, and the median overall survival was 5.0 and 4.0 months, respectively. Cum, cumulative.

CEA and CA199, tumor markers in the gastrointestinal tract, provide a reference for the diagnosis of duodenal tumor.

Duodenal papilla carcinoma is a rare tumor. Duodenal papilla carcinoma accounts for $<1 \%$ of all digestive system malignant tumors. Duodenal papilla cancer, due to the special location of the lesion, usually causes an obstruction to an early diagnosis with respect to biliary tract symptoms, and the clinical manifestations of progressive painless jaundice. In the present study, it has been demonstrated that the $\mathrm{N}$ and $M$ stages of adenocarcinoma of the duodenal papilla occurred earlier compared with those of the non-duodenal papilla, and this could be associated with the influence of the jaundice particularly associated with adenocarcinoma of the duodenal papilla.

The treatment of duodenal adenocarcinoma largely depends on surgical resection. The present study has also revealed that radical surgery was more effective compared with other treatments. Surgical procedures include pancreatoduodenectomy, duodenal segment resection, 
Table VIII. Treatment options.

\begin{tabular}{lccc}
\hline & & Number of patients \\
\cline { 2 - 4 } & Surgery & $\begin{array}{c}\text { Surgery plus } \\
\text { chemotherapy }\end{array}$ & $\begin{array}{c}\text { Supportive } \\
\text { therapy }\end{array}$ \\
\hline $\begin{array}{l}\text { Non-ampulla } \\
\text { Ampulla }\end{array}$ & 8 & 2 & 11 \\
\hline
\end{tabular}

Statistical analysis: $\chi^{2}=3.473, v=2, \mathrm{P}=0.176$ (two-sided). The difference was not significant. There was no significant difference in the choice of treatment for patients with the ampulla or the non-ampulla duodenal tumor.

Table IX. Operation mode.

\begin{tabular}{|c|c|c|c|c|}
\hline & \multicolumn{4}{|c|}{ Number of patients } \\
\hline & Pancreatoduodenectomy & $\begin{array}{l}\text { Partial duodenum } \\
\quad \text { resection }\end{array}$ & $\begin{array}{c}\text { Billroth II } \\
\text { subtotal gastrectomy }\end{array}$ & $\begin{array}{l}\text { Palliative } \\
\text { operation }\end{array}$ \\
\hline Non-ampulla & 3 & 3 & 2 & 2 \\
\hline Ampulla & 12 & 0 & 0 & 5 \\
\hline
\end{tabular}

Table X. The survival time of different treatment for patients with adenocarcinoma (month).

\begin{tabular}{ll}
\hline Treatment & \multicolumn{1}{c}{ Survival time (months) } \\
\hline Radical surgery (no chemotherapy) & $9,40.6,18+, 33+, 4.7,12.5,12-, 60,50,35+, 7,25$ \\
Radical surgery + chemotherapy & $71,26.8,15,19.7,181,30+$ \\
Supportive therapy (no chemotherapy) & $3,3,0.7,6,4.8,2.5,6,1,12-, 4,6,0.4,2,8,10,12-$ \\
Palliative therapy (no chemotherapy) & $5,13,11.4,1.3,1,1$ \\
Palliative therapy + chemotherapy & 25.4 \\
\hline
\end{tabular}

Plus or minus symbols following after the data, where shown, is indicative of the fact that these data are censored.

palliative bypass surgery, and so forth. The choice of surgical approach depends predominantly on the location of the tumor, tumor staging, the patient's general situation and the level of experience. If the patient's health status is good, radical resection of the duodenum is the first choice for the treatment of duodenal papilla carcinoma (11). A number of surgeons also consider that duodenal papillary tumor local excision embodies the principles of modern, minimally invasive surgery after the resection of tumors, and to the greatest extent this: i) leads to a retention of normal levels of human bile and pancreatic juice; and ii) is mainly applicable to duodenal papilla in patients with benign tumors and for patients with malignant tumors where a pancreatoduodenectomy could not be tolerated; therefore, this method is gaining in popularity in the clinic (12). In the present study, the use of pancreatoduodenectomy for duodenal papilla carcinoma was more reliable. In our group of 12 patients with duodenal papilla carcinoma who underwent radical operation, three patients experienced long-term survival. Radical resection of duodenal cancer is the most effective method of treatment; pancreatoduodenectomy is preferred as the method of choice for the tumor area around the nipple, and it may also be used in duodenum primary adenocarcinoma. For adenocarcinoma located in segments 3 or 4 of the duodenum, with no local lymph node metastasis, and where the patients are in poor general condition and are unable to tolerate large-scale surgery, duodenal segment resection may be an option. There are reports that the two types of operation are not significantly different, suggesting that duodenal segment resection should be the first choice for adenocarcinoma located in segments 3 or 4 of the duodenum (13). For patients with advanced cancer tumors that are not able to be resected, feasibly, palliative bypass surgery can be implemented, for example, a stomach jejunum anastomosis, or internal and external drainage of the bile duct. Based on the present study, our analysis indicates 
that pancreatoduodenectomy would be a radical operation for duodenal papilla carcinoma located in the ampulla. However, for non-ampulla duodenal gland cancer, duodenum partial resection or subtotal gastrectomy may also be able to achieve the radical goal.

The majority of commentators suggest that adjuvant radiotherapy and chemotherapy are able to improve the prognosis of duodenal adenocarcinoma, although it has been reported that adjuvant chemotherapy cannot improve the prognosis of patients (14). In our study, there was no survival benefit for adjuvant chemotherapy in duodenal adenocarcinoma. In conclusion, the incidence of duodenal adenocarcinoma is low, the diagnosis is difficult and the prognosis is poor. Early diagnosis and radical resection are the key to an improved prognosis.

Gastrointestinal stromal tumor (GIST) is a type of non-directional differentiation tumor that independently originates from the primitive mesenchymal tissues of the gastrointestinal tract. Duodenal stromal tumors are derived in Cajal interstitial cells or more primitive mesoderm mesenchymal stem cells with multipotential differentiation, and these are clinically rare. The incidence of GIST is $\sim 1-2$ per 200,000 , and it may occur across the length of the entire digestive tract, from the esophagus to the anus, also including the retina, the mesentery, the peritoneum and the retroperitoneal area. Most commonly however, it occurs in the stomach and the small intestine, while duodenal stromal tumors are less frequent, accounting for only $10 \%$ of the small intestine (15). Duodenal stromal tumors are predominantly concentrated in the descending and horizontal portion of the duodenum, and less so in the bulb and the ascending portion (16). Certain researchers have reported that recurrent black stool is the most common clinical symptom of duodenal stromal tumor (17). The invasion of the stroma is not strong, rarely leading to obstruction of the bile duct or the pancreatic duct. In our study, of 44 cases of duodenal tumor, only two cases were stromal tumors, both of which were located in the non-ampulla duodenum.

Surgical resection is the first choice for treatment of duodenal stromal tumors, and the goal is to achieve the $\mathrm{R} 0$ removal of negative tissue margins. Common operational techniques include partial duodenum resection, pancreatoduodenectomy, distal subtotal gastrectomy and duodenal segment resection. A study has shown that the incidence of postoperative complications associated with pancreatoduodenectomy was greater compared with the other surgical procedures, and an expanded resection range could not improve a survival rate of 5 years following the surgery (18). Therefore, considered from two different aspects, namely, to ensure the safety of the surgery and to improve the survival time, blind implementation of pancreatoduodenectomy should be avoided. Pancreaticoduodenectomy is suitable for tumors of diameter $\geq 5 \mathrm{~cm}$ located in the bulb or the descending portion of the duodenum, where there is duodenal nipple involvement, or in cases of recurrence after surgery. Usually, GISTs rarely occur in lymph node metastasis, and it is thought that, in duodenal stromal tumor surgery, unless there is evidence to support such a practice, cleaning of the lymph nodes is not required (19). For stromal tumors where there is more expansive growth or rare lymph node metastasis, excessive expansion excision would not prolong the survival time of the patients. In a study that advocated ensuring a negative margin necessary to perform the required surgical resection, a wide range of lymph node dissection was not advocated (20). In our study, there were two cases of non-ampullary duodenal tumors. One case was located in the descending part, and the patient refused an operation. One case was located in the horizontal section, and partial resection of the duodenum was performed; the pathological stage was III, $\mathrm{PT}_{3} \mathrm{G}_{1} \mathrm{M}_{0}$. This patient has survived for more than 10 years, and the operation therefore effected a radical cure.

Our study also identified one case of duodenal large B-cell lymphoma, which is clinically rare and has not previously reported by others in the literature, which was categorized as gastrointestinal lymphoma, clinical stage II E period. The patient underwent a Billroth II subtotal gastrectomy, and the patient has survived for 3 years following the operation.

Primary melanoma of the duodenum has also been reported in the literature (21), although it was not identified in the present study.

In conclusion, the present study has shown that duodenal tumors are a rare disease, although they accounted for the vast majority of small intestinal tumors. The pathological type was mainly adenocarcinoma; periampullary duodenal tumors were nearly all adenocarcinoma. Non-ampulla duodenal tumors also included rare pathological types, such as stromal tumors and large B-cell lymphoma, in addition to predominantly adenocarcinoma. The clinical manifestations of duodenal tumor were not specific compared with other diseases of the digestive tract, leading to difficult early diagnosis and treatment. Due to the clinical manifestation of jaundice, periampullary duodenal tumors were diagnosed at an earlier stage compared with non-ampulla duodenal tumors. Endoscopy and CT examination are valuable in diagnosis, and may be used as a means of screening. CEA and CA199 also are very important in terms of diagnosis. Radical surgery is the most effective treatment, and pancreaticoduodenectomy applicable for all duodenal tumors. For non-ampulla duodenal tumors, partial duodenum resection and subtotal gastrectomy may be selected. No survival benefit was identified for adjuvant chemotherapy. It is expected that there will be further, larger scale retrospective studies to further explore the relevant issues.

\section{Acknowledgements}

We would like to thank, with their permission, our colleagues, W.J.R., L.S.J. and H.J.S., who assisted in or collaborated with us in this study, and who did not meet with the criteria for full authorship. No sources of funding were available for any of authors.

\section{References}

1. Chen J-R, Zhang Y-Q and Chen K-N: Imaging diagnosis of intestinal tumors. Chinese Medical Computer Imaging Journal 7: 93-102, 2001

2. Cortese AF and Cornell GN: Carcinoma of the duodenum. Cancer 29: 1010-1015, 1972.

3. Xia R-M, Zhang S-Z and Zhang H-L: CT diagnosis and differential diagnosis of duodenal malignant tumors. Pract Radiol J 21: 48-50, 2005.

4. Hu F and Ma Y-J: Diagnosis and treatment experience of 30 cases primary malignant duodenal tumors. Chin Med J 22: 41-42, 2009. 
5. Ge X-B: Comparative study of X-ray and CT diagnosis of duodenal adenocarcinoma. Chin CT and MRI J 12: 84-86, 2014.

6. Liang L, Ma L, Xiao Y, Zhang Y and Li H-Y: Clinical analysis of 55 cases of primary small bowel malignant tumor. Tumor 29: 589-591, 2009

7. Zhang S-S, Sun D-L, Liu D, Li J-M and Chen L: Clinical analysis of 56 cases of primary duodenal adenocarcinoma. Chin J Gen Surg 20: 534-535, 2005.

8. He Y-N, Ye M-X and Lei Z-M: Analysis of 77 cases of primary duodenal tumors. Diagnosis \& Treatment of Cancer Prevention \& Treatment 24: 154-156, 2011.

9. He Z-H: Comparison of detection rate of four methods of duodenal papilla carcinoma (24 cases analysis). J Pract Oncol 19: 347-348, 2004

10. Tang Z-Y, Wang Y-F, Chen Y-R, Zhou E-H and Shan X-H: Value of $\mathrm{CT}$ in the diagnosis of primary duodenal malignant tumors. Radiol Pract 27: 880-884, 2012.

11. Zhou F, Huang M-W, Xu Z, Luo Z-Q, Zhou S-B, Shao J-H and Wang K: Application and evaluation of Laparoscopic assisted pancreaticoduodenectomy in the treatment of duodenal papilla carcinoma. Chin J Clin Oncol 38: 166-169, 2011.

12. Wang J and Yang L-H: Local excision of duodenal papilla tumor. Chin J Digest Surg 12: 520-523, 2013

13. Zhang S-S, Chen L, Leng X-S, Zhang X-F and Shao Y-F: Clinical analysis of 89 cases of duodenal adenocarcinoma. Chin J Gen Surg 26: 543-545, 2011

14. Struck A, Howard T, Chiorean EG, Clarke JM, Riffenburgh R and Cardenes HR: Non-ampullary duodenal adenocarcinoma: Factors important for relapse and survival. J Surg Oncol 100: $144-148,2009$
15. Yildirgan MI, Basoghu M, Atamanalp SS, Albayrak Y, Gürsan N and Onbaş O: Duodenal stromal tumor: Report of a case. Surg Today 37: 426-429, 2007.

16. Chen Q-N, Peng C-H, Wang J-C, Han B-S, Cheng D-F, Lu Z and Wang X-M: Clinical analysis of 16 cases of duodenal stromal tumors. J Hepatobiliary Surg 15: 108-110, 2007.

17. Jin X-B and Wang D-Q: Experience of diagnosis and treatment of 12 cases of duodenal stromal tumors. Pract Med 24: 1009-1010, 2008.

18. Lu Z-H, Wu X-J, Fang Y-J, Pan Z-Z and Wan D-S: Surgical treatment and prognosis of gastrointestinal stromal tumor. Chin J Gastrointestinal Surg 14: 778-780, 2011.

19. Li Y-J, Zhou J-P, Kong F-M, Tian Y-L and Dong M: Operation mode study of duodenal stromal tumors and literature review of 219 cases. Shandong Medicine 54: 85-86, 2014.

20. Lanuke K, Bathe OF and Mack LA: Local excision of duodenal gastrointestinal stromal tumor. J Surg Oncol 95: 267-269, 2007.

21. Korkolis DP, Apostolaki K, Gontikakis E, Plataniotis GD, Siskos D, Xinopoulos D, Dimitroulopoulos D, Papantoniou N, Biteli $\mathrm{M}$ and Vassilopoulos PP: Primary malignant melanoma of the duodenum: Aggressive management and long-term survival of an unusual oncologic entity. South Med J 101: 836-839, 2008. http://doodle.com/poll/9533dgd7in3ebd5e\# 